\title{
ISOMORPHISMS OF HYPERGROUPS
}

\author{
WALTER R. BLOOM and MARTIN E. WALTER
}

(Received 14 August 1990)

Communicated by W. Moran

\begin{abstract}
Let $K_{1}, K_{2}$ be locally compact hypergroups. It is shown that every isometric isomorphism between their measure algebras restricts to an isometric isomorphism between their $L^{1}$-algebras. This result is used to relate isometries of the measure algebras to homeomorphisms of the underlying locally compact spaces.
\end{abstract}

1991 Mathematics subject classification (Amer. Math. Soc.): primary 43 A 62; secondary 43 A10, 43 A 20, 43 A 22.

During the past forty years there have been many results characterising locally compact groups in terms of spaces of functions and measures on them. The aim has been to find complete invariants of the class of locally compact groups in the following sense: the locally compact groups $G_{1}, G_{2}$ are topologically isomorphic if and only if the corresponding function or measure spaces $E\left(G_{1}\right), E\left(G_{2}\right)$ are isomorphic in the appropriate category. In this context, the development for $L^{1}(G)$ began with the papers of Kawada (1948) and Wendel $(1951,1952)$; for $M^{1}(G)$ with Johnson (1964) and Strichartz (1965), and for $A(G)$ and $B(G)$ with Arendt and Cannière (1983) and Walter (1972). There have been various extensions to spaces of continuous functions and $p$ th-integrable functions, and also variations on the nature of the isomorphism between $E\left(G_{1}\right)$ and $E\left(G_{2}\right)$.

In this paper we consider the above ideas in the hypergroup setting. A hypergroup (which we define in detail in Section 1 ) is a locally compact space $K$ with a convolution on its measure space $M(K)$ such that $(M(K),+, *)$

(C) 1992 Australian Mathematical Society $0263-6115 / 92 \$ A 2.00+0.00$ 
is a Banach algebra with further convolution-like properties. Familiar examples include the space of conjugacy classes of a compact group, the space of double cosets of a locally compact group, and the dual object of a compact group. It is important to adopt the viewpoint that hypergroups have no algebraic structure of their own; all properties are inherited through the measure algebra. As such, complete invariants need to be given in terms of the measure algebra itself.

Notwithstanding the above, we can give useful analogues of the Kawada/ Wendel and Johnson/Strichartz results, by showing that these are in fact statements about the convolution measure algebra structures rather than being inexorably tied to the underlying group.

This paper is presented in four sections. In the first we give a definition of a hypergroup and present some preliminary results. Section 2 will be concerned with the Kawada/Wendel result, where we identify the isometric isomorphisms of the (hyper)group algebra $L^{1}(K)$ in terms of bipositive isomorphisms. In Section 3 we show that isometric isomorphisms between hypergroup algebras are determined by isometric isomorphisms between the corresponding measure algebras, thus extending the results of Johnson (1964) and Strichartz (1965) given for locally compact groups. We also prove the converse of this. Finally in Section 4 we show how isomorphisms between measure algebras lead to homeomorphisms and other structure preserving mappings between the underlying locally compact spaces.

\section{Hypergroups}

Hypergroups made their first appearance in harmonic analysis with the papers of Dunkl (1973), Jewett (1975) and Spector (1975). While the three definitions differ slightly the developments are quite similar. We follow that of Jewett, which has become the standard for the many papers appearing in this area during the ensuing fifteen years.

1.1. Notation. Let $X$ be a locally compact Hausdorff space. The notation below will be used throughout the paper.

$\mathbf{R}, \mathbf{C}$

$C(X), C_{0}(X), C_{c}(X)$

$M(X)$

$M^{+}(X), M^{1}(X)$
Space of real and complex numbers, respectively.

Space of bounded continuous complex-valued functions on $X$, those vanishing at infinity, and those with compact support, respectively. Space of bounded Radon measures on $X$. Subset of $M(X)$ consisting of those measures 
that are nonnegative, and those that are nonnegative with total variation one, respectively.

$\operatorname{supp}(\mu) \quad$ Support of $\mu \in M(X)$.

$\operatorname{supp}(A) \bigcup\{\operatorname{supp}(\mu): \mu \in A\}$, where $A \subset M(X)$.

$\|\mu\| \quad$ Total variation norm of $\mu \in M(X)$.

$\varepsilon_{x} \quad$ Point mass at $x$.

$A^{c} \quad$ Closure of $A \subset X$.

$\xi_{A} \quad$ Indicator function of $A \subset X$.

$\varnothing \quad$ Empty set.

$\bar{A} \quad$ Complex conjugate of $A \subset C$.

In this paper we consider two topologies on $M(X)$, namely the vague topology $\sigma\left(M(X), C_{c}(X)\right)$, and $\sigma(M(X), C(X))$ which we refer to as the weak topology (Jewett (1975) uses the term cone topology). Where nothing is said to the contrary, it is the weak topology that is intended.

1.2. Hypergroups. A nonvoid locally compact Hausdorff space $K$ will be called a hypergroup if the following conditions are satisfied.

(i) $(M(K),+)$ admits a binary operation $*$ under which it is a complex algebra.

(ii) The bilinear mapping $*: M(K) \times M(K) \rightarrow M(K)$ given by $(\mu, \nu) \rightarrow$ $\mu * \nu$ is nonnegative $(\mu * \nu \geq 0$ whenever $\mu, \nu \geq 0)$ and its restriction to $M^{+}(K) \times M^{+}(K)$ is continuous when $M^{+}(K)$ is given the weak topology.

(iii) Given $x, y \in K, \varepsilon_{x} * \varepsilon_{y} \in M^{1}(K)$ and $\operatorname{supp}\left(\varepsilon_{x} * \varepsilon_{y}\right)$ is compact.

(iv) The mapping $(x, y) \rightarrow \operatorname{supp}\left(\varepsilon_{x} * \varepsilon_{y}\right)$ of $K \times K$ into the space of nonvoid compact subsets of $K$ is continuous, the latter space with the topology as given in Jewett (1975), Section 2.5.

(v) There exists a (necessarily unique) element $e$ of $K$ such that $\varepsilon_{e} * \varepsilon_{x}=$ $\varepsilon_{x} * \varepsilon_{e}=\varepsilon_{x}$ for all $x \in K$.

(vi) There exists a (necessarily unique) involution (a homeomorphism $x \rightarrow x^{-}$of $K$ onto itself with the property $\left(x^{-}\right)^{-}=x$ for all $x \in K)$ such that, for $x, y \in K, e \in \operatorname{supp}\left(\varepsilon_{x} * \varepsilon_{y}\right)$ if and only if $x=y^{-}$, and $(\mu * \nu)^{-}=\nu^{-} * \mu^{-}$for all $\mu, \nu \in M(K)$, where $\mu^{-} \in M(K)$ is defined by $\mu^{-}(A)=\mu\left(A^{-}\right)$for Borel subsets $A$ of $K$, with $A^{-}=\left\{x^{-}: x \in A\right\}$.

A familiar example of a hypergroup is a locally compact Hausdorff group $G$, with $M(G)$ carrying its usual convolution structure. Many examples are to be found in Dunkl (1973), Jewett (1975), Ross (1977) and Bloom and Heyer (1982). Harmonic analysis on hypergroups has been given in some detail in the paper of Jewett (1975). We shall use the more straightforward 
results there without explicit reference. However some of the results that either we need to refer to reasonably often or are not to be found in the form we require will be presented below.

For $f \in C(K), x, y \in K$, we write

$$
f(x * y)=\int f d\left(\varepsilon_{x} * \varepsilon_{y}\right)=f_{x}(y)=f^{y}(x)
$$

provided the integral exists. It is seen that $(x, y) \rightarrow f(x * y)$ is a continuous function on $K \times K$, and that $f_{x}, f^{x} \in C(K)$ for each $x \in K$.

For subsets $A, B$ of $K$ define

$$
A * B=\bigcup\left\{\operatorname{supp}\left(\varepsilon_{x} * \varepsilon_{y}\right): x \in A, y \in B\right\} .
$$

A subset $H$ of $K$ is called a subhypergroup of $K$ if the following three conditions are satisfied:

(i) $H$ is nonvoid and closed;

(ii) $H^{-}=H$;

(iii) $H * H \subset H$.

It is apparent that $e \in H$ whenever $H$ is a subhypergroup of $K$.

Let $H$ be a subhypergroup of $K$. A nonnegative (not necessarily bounded) Radon measure $\omega$ on $K$ will be called left $H$-invariant if $\varepsilon_{x} * \omega=\omega$ for all $x \in H$. In the case where $\omega$ is left $K$-invariant, we refer to it as a left Haar measure on $K$. If $K$ is compact (Jewett (1975), Theorem 7.2A), discrete (Jewett (1975), Theorem 7.1A) or commutative (Spector (1978), Theorem III.4) then $K$ possesses a left Haar measure. It is not known whether all hypergroups admit left Haar measures. However if a left Haar measure exists, it is essentially unique (Jewett (1975), Theorem 5.2). Throughout this paper we assume that $K$ possesses a left Haar measure, which we denote by $\omega_{K}$, or $\omega$ if there is no possible ambiguity. We denote by $M_{a}(K)$ the two-sided ideal in $M(K)$ consisting of those measures absolutely continuous with respect to $\omega$; see Jewett (1975), Theorem 5.6A. Similar considerations hold when left is replaced by right throughout. A measure that is both left and right $\mathrm{H}$ invariant will be referred to simply as $H$-invariant. It should be noted that a left Haar measure on a compact hypergroup $K$ is also right $K$-invariant (Jewett (1975), Theorem 7.2A).

Of particular importance in probability theory are those measures $\omega \in$ $M^{+}(K)$ satisfying $\omega * \omega=\omega$; such measures are called idempotent. Jewett (1975), Theorem 10.2E has characterised the idempotent measures as Haar measures of compact subhypergroups of $K$; see also Dunkl (1973), Theorem 1.13 .

For each $f \in C(K)$ we define $f^{-}$by $f^{-}(x)=f\left(x^{-}\right)$, where $x \in K$. It is clear that $\int f^{-} d \mu=\int f d \mu^{-}$whenever $\mu \in M(K)$. Furthermore 
$\underline{(M(K)},+, *)$ is a Banach $\sim$-algebra, with the involution given by $\mu^{\sim}(A)=$ $\overline{\mu\left(A^{-}\right)}$. For each $p \in[1, \infty]$ we can form the Lebesgue space $L^{p}(K, \omega)$ which is usually written just as $L^{p}(K)$. We shall not distinguish between $L^{1}(K)$ and its isomorphic image $M_{a}(K)$ in $M(K)$.

\section{Isomorphisms of $L^{1}(K)$}

Let $T$ be an isometric isomorphism between $L^{1}\left(K_{1}\right)$ and $L^{1}\left(K_{2}\right)$, and write $s(f)=\{x \in K: f(x) \neq 0\}$ where $f \in L^{1}(K)$. (This support set is determined up to a null set.) We need the following five lemmas which were given by Wendel (1951) for locally compact groups. However it should be noted that the proofs given by Wendel for Lemmas 2.1-2.4 do not depend on any group structure; the reader is referred to Wendel (1951), pp. 308-309 for these.

2.1. Lemma. For $f, g \in L^{1}\left(K_{1}\right), s(f) \cap s(g)=\varnothing$ if and only if $s(T f) \cap$ $s(T g)=\varnothing$.

2.2. Lemma. For $f, g \in L^{1}\left(K_{1}\right), s(f) \subset s(g)$ if and only if $s(T f) \subset$ $s(T g)$.

2.3. LemMa. For each $\sigma$-finite subset $B$ of $K_{2}$ there exists $f \in L^{1}\left(K_{1}\right)^{+}$ such that $s(T f)=B$.

2.4. Lemma. Let $f, g \in L^{1}\left(K_{1}\right)^{+}$. For $y \in s(T f)$ write

$$
K_{T f}(y)=T f(y)|T f(y)|^{-1}
$$

and similarly for $K_{T g}$. Then $K_{T f}=K_{T g}$ a.e. on $s(T f) \cap s(T g)$.

The fifth lemma is essential for relating isometric and positive isomorphisms between the hypergroup algebras. We first require the idea of a continuous character $\gamma$, which is a nontrivial bounded continuous function satisfying both the product formula $\gamma(x * y)=\gamma(x) \gamma(y)$ and $\gamma\left(x^{-}\right)=\overline{\gamma(x)}$ for all $x, y \in K$. We write $K^{\wedge}$ for the set of continuous characters on $K$. Note that members of $K^{\wedge}$ are necessarily bounded in modulus by $\gamma(e)=1$ (see Jewett (1975), Lemma 6.3D) but in contrast to the group case could take the value zero. 
2.5. LEMMA. There is a unique continuous character $\gamma$ with $|\gamma|=1$ on $K_{2}$ such that $T f=\gamma|T f|$ a.e. on $K_{2}$ for all $f \in L^{1}\left(K_{1}\right)^{+} \quad(\gamma$ is real or complex according to the $L^{1}$-spaces).

Proof. Let $V$ be a compact neighbourhood of $e_{2} \in K_{2}$, and using Jewett (1975), Lemma 10.1C choose an open (and closed) $\sigma$-compact subhypergroup $H_{0}$ of $K_{2}$ containing $V$. By Lemma 2.3 there exists $h \in L^{1}\left(K_{1}\right)^{+}$ such that $s(T h)=H_{0}$. From $h \geq 0$ we have $\|h * h\|_{1}=\|h\|_{1}^{2}$ so that

$$
\|T h * T h\|_{1}=\|T(h * h)\|_{1}=\|h * h\|_{1}=\|h\|_{1}^{2}=\|T h\|_{1}^{2} .
$$

Now

$$
T h * T h(y)=\int_{H_{0}} T h(z) T h\left(z^{-} * y\right) d \omega_{K_{2}}(z) .
$$

Using Lemma 2.4, we have (writing $K$ for the common value)

$$
K=K_{T h * T h}=K_{T h} \quad \text { a.e. on } s(T h * T h) \cap s(T h) .
$$

As $s(T h)=H_{0}$ is a subhypergroup of $K_{2}, s(T h * T h) \subset H_{0}$ so that $s(T h * T h) \subset s(T h)$. Now as $T h * T h=0$ outside $s(T h * T h)$ we have that $T h * T h=K|T h * T h|$ on $H_{0}$, where we have extended $K$ to be $K_{T f}$ on $H_{0}$. Hence

$$
|T h * T h(y)|=K(y)^{-1} \int_{H_{0}} T h(z) T h\left(z^{-} * y\right) d \omega_{K_{2}}(z)
$$

and using (1), we have

$$
\begin{aligned}
\|T h * T h\|_{1} & =\int_{H_{0}} K(y)^{-1} \int_{H_{0}} T h(z) T h\left(z^{-} * y\right) d \omega_{K_{2}}(z) d \omega_{K_{2}}(y) \\
& =\int_{H_{0}} \int_{H_{0}} K(y)^{-1} K(z)|T h|(z)(K|T h|)\left(z^{-} * y\right) d \omega_{K_{2}}(z) d \omega_{K_{2}}(y) \\
& =\|T h\|_{1}^{2} \\
& =\int_{H_{0}}|T h|(y) d \omega_{K_{2}}(y) \int_{H_{0}}|T h|(z) d \omega_{K_{2}}(z) \\
& =\int_{H_{0}} \int_{H_{0}}|T h|(z)|T h|\left(z^{-} * y\right) d \omega_{K_{2}}(y) d \omega_{K_{2}}(z)
\end{aligned}
$$

where for the final equality we have used Jewett (1975), Theorem 3.3F. It follows that as $\varepsilon_{z^{-}} * \varepsilon_{y}$ is supported in $H_{0}$

$$
\begin{gathered}
\int_{H_{0}} \int_{H_{0}} \int_{\left\{z^{-}\right\} *\{y\}} K(x) K(y)^{-1} K(z)|T h|(x)|T h|(z) \\
d \varepsilon_{z^{-}} * \varepsilon_{y}(x) d \omega_{K_{2}}(z) d \omega_{K_{2}}(y) \\
=\int_{H_{0}} \int_{H_{0}} \int_{\left\{z^{-}\right\} *\{y\}}|T h|(x)|T h|(z) d \varepsilon_{z^{-}} * \varepsilon_{y}(x) d \omega_{K_{2}}(z) d \omega_{K_{2}}(y) .
\end{gathered}
$$


Since $\left|K(x) K(y)^{-1} K(z)\right|=1$ we deduce that $K(x)=K(y) K(z)^{-1}$ with the equalities holding $x$-a.e. on $\left\{z^{-}\right\} *\{y\}$ for almost all $y, z$. Hence

$$
\int_{\left\{z^{-}\right\} *\{y\}} K(x) d \varepsilon_{z^{-}} * \varepsilon_{y}(x)=K(y) K(z)^{-1}
$$

and $K\left(z^{-} * y\right)=K(y) K(z)^{-1}$ for almost all $y, z \in H_{0}$. Now choose a compact set $C \subset H_{0}$ of (finite) positive measure, and integrate to obtain

$$
K(y) \omega_{K_{2}}(C)=\int_{C} K(y) d \omega_{K_{2}}(z)=\int_{C} K\left(z^{-} * y\right) K(z) d \omega_{K_{2}}(z)
$$

for almost all $y$. Since $K \in L^{\infty}\left(K_{2}\right)$ and $K \xi_{c} \in L^{1}\left(K_{2}\right)$, Jewett (1975), Theorem 5.5D shows that the latter expression is a continuous function of $y$. Thus $K$ is equal almost everywhere to a continuous function, $\gamma_{0}$ say. By the above $\gamma_{0}\left(z^{-} * y\right)=\gamma_{0}(y) \gamma_{0}(z)^{-1}$ a.e. on $H_{0} \times H_{0}$. Appealing to Jewett (1975), Lemma 3.1A, we see that this equality holds everywhere on $H_{0} \times H_{0}$, and it should also be observed that $\left|\gamma_{0}\right|=1$.

We note from Lemma 2.4 that for any $f \in L^{1}\left(K_{1}\right)^{+}$with $s(T f) \subset H_{0}$, $T f=\gamma_{0}|T f|$ a.e. on $H_{0}$. We now extend this result to all of $K_{2}$. Write $K_{2}=\bigcup_{\alpha}\left\{y_{\alpha}\right\} * H_{0}$ as the disjoint union of left hypercosets of $H_{0}$ (see Jewett (1975), Lemma 10.3A), pick a finite number of them, and write $H_{1}$ for the open subhypergroup of $K_{2}$ generated by the (finite) union. By Jewett (1975), Theorem $10.1 \mathrm{C}, H_{1}$ is $\sigma$-compact, and as above we obtain a continuous character $\gamma_{1}$ with modulus 1 on $H_{1}$. By Lemma 2.4 for two such subhypergroups $H_{1}, H_{1}^{\prime}, \gamma_{1}$ and $\gamma_{1}^{\prime}$ agree on $H_{1} \cap H_{1}^{\prime} \supset H_{0}$ so that $\gamma_{1}$ is an extension of $\gamma_{0}$. It is easy to see that for any $f \in L^{1}\left(K_{1}\right)^{+}$with $s(T f) \subset H_{1}$, $T f=\gamma_{1}|T f|$ a.e. on $H_{1}$.

Now extend $\gamma_{0}$ to $\gamma$ on $K_{2}$ which by the preceding paragraph is well defined. Consider any $f \in L^{1}\left(K_{1}\right)^{+}$, and since $\operatorname{supp}\left(\omega_{1}\right)=K_{1}$ (Jewett (1975), Lemma 5.1A) the countable number of hypercosets $\left\{y_{n}\right\} * H_{0}$ that $s(T f)$ intersects nontrivially (that is, in sets of positive measure). Write

$$
g_{n}=\left.T f\right|_{\left\{y_{n}\right\} * H_{0}}
$$

and $f_{n}=T^{-1} g_{n}$. Then $f=\sum_{n=1}^{\infty} f_{n}$, and by Lemma 2.1 the sets $s\left(f_{n}\right)$ are pairwise disjoint. Since $f \geq 0$ so is each $f_{n}$. Thus

$$
g_{n}(y)=\gamma_{n}(y)\left|g_{n}(y)\right|=\gamma(y)\left|g_{n}(y)\right|, \quad y \in\left\{y_{n}\right\} * H_{0},
$$

and

$$
T f(y)=\gamma(y)|T f(y)| \quad \text { for all } y \in K_{2} .
$$

We can now present the main result of this section. 
2.6. TheOREM. Let $T$ be an isometric isomorphism of $L^{1}\left(K_{1}\right)$ onto $L^{1}\left(K_{2}\right)$. There is a continuous character $\gamma$ on $K_{2}$ with modulus 1 such that the mapping $P: L^{1}\left(K_{1}\right) \rightarrow L^{1}\left(K_{2}\right)$ defined by

$$
(P f)(x)=\gamma(x)^{-1} T f(x), \quad f \in L^{1}\left(K_{1}\right), x \in K_{2},
$$

is an isomorphism of $L^{1}\left(K_{1}\right)$ onto $L^{1}\left(K_{2}\right)$ satisfying $P f \geq 0$ if and only if $f \geq 0$. The character $\gamma$ is real or complex according to $L^{1}\left(K_{1}\right), L^{1}\left(K_{2}\right)$.

Proof. By Lemma 2.5 there is a (unique) continuous character $\gamma$ of $K_{2}$ with $|\gamma|=1$ such that for all $f \in L^{1}\left(K_{1}\right)^{+}, T f=\gamma|T f|$ a.e. on $K_{2}$. With this choice of $\gamma$ define $P$ as in the statement of the theorem, and for $f \geq 0$

$$
(P f)(x)=\gamma(x)^{-1} T f(x)=|T f(x)|
$$

so that $P$ carries positive elements of $L^{1}\left(K_{1}\right)$ into positive elements of $L^{1}\left(K_{2}\right)$.

We also have from the proof of the lemma in Bloom (1986) that for $f, g \in$ $L^{1}\left(K_{1}\right)$

$$
P(f * g)=\gamma^{-1} T(f * g)=\gamma^{-1}(T f * T g)=\left(\gamma^{-1} T f\right) *\left(\gamma^{-1} T g\right)=P f * P g
$$

(note that the lemma is given for commutative hypergroups, but the proof carries over to the general case by appealing to Dunkl (1973), Proposition 2.2(3) where it is shown that $\gamma$ is constant with value $\gamma(x) \gamma(y)$ on $\{x\} *\{y\})$. Thus $P$ is an algebra isomorphism of $L^{1}\left(K_{1}\right)$ onto $L^{1}\left(K_{2}\right)$.

It remains to show that $f \geq 0$ whenever $P f \geq 0$. Write

$$
f=f_{1}-f_{2}+i\left(f_{3}-f_{4}\right)
$$

where $f_{1}, f_{2}, f_{3}, f_{4} \geq 0$ and $s\left(f_{1}\right) \cap s\left(f_{2}\right)=\varnothing=s\left(f_{3}\right) \cap s\left(f_{4}\right)$. Then

$$
P f=P f_{1}-P f_{2}+i\left(P f_{3}-P f_{4}\right)
$$

and since $P$ is an isometry Lemma 2.1 implies that

$$
s\left(P f_{1}\right) \cap s\left(P f_{2}\right)=\varnothing=s\left(P f_{3}\right) \cap s\left(P f_{4}\right) .
$$

Now $P f \geq 0$ implies that $P f_{3}-P f_{4}=0$, and using the disjointness of the supports $P f_{2}=P f_{3}=P f_{4}=0$ (all three terms are nonnegative). Thus $P f=P f_{1}$ and $f=f_{1} \geq 0$ as required.

The algebra isomorphism $P$ introduced in Theorem 2.6 is termed bipositive. We have a converse to Theorem 2.6 for such operators. 
2.7. THEOREM. Let $P$ be a bipositive isomorphism of $L_{R}^{1}\left(K_{1}\right)$ onto $L_{R}^{1}\left(K_{2}\right)$. Then $P$ is an isometry.

Proof. $P$ and $P^{-1}$ are order-preserving hence bounded. Thus as in Wendel (1951), Theorem 2

$$
0<C_{1} \leq\|P f\|_{1} /\|f\|_{1} \leq C_{2}<\infty, \quad f \neq 0 .
$$

Let $f \in L^{1}\left(K_{1}\right)^{+}$. Then Jewett (1975), Theorem 5.5L shows that $\|f * f\|_{1}=$ $\|f\|_{1}^{2}$ and by recursion $\left\|f^{* n}\right\|_{1}=\|f\|_{1}^{n}$ for all $n=1,2, \ldots$. Now $P f \geq 0$ and $P\left(f^{* n}\right)=(P f)^{* n}$, so that by (2)

$$
0<C_{1} \leq\left(\|P f\|_{1} /\|f\|_{1}\right)^{n} \leq C_{2}<\infty, \quad f \neq 0, \quad n=1,2, \ldots
$$

Thus $\|P f\|_{1}=\|f\|_{1}$ for all $f \in L^{1}\left(K_{1}\right)^{+}$.

For $f \in L_{R}^{1}\left(K_{1}\right)$ write $f=f_{1}-f_{2}$ where $f_{1}, f_{2}$ are nonnegative with disjoint supports. Then

$$
\begin{aligned}
\|f\|_{1}=\left\|f_{1}-f_{2}\right\|_{1} & =\left\|f_{1}\right\|_{1}+\left\|f_{2}\right\|_{1}=\left\|P f_{1}\right\|_{1}+\left\|P f_{2}\right\|_{1} \\
& \geq\left\|P f_{1}-P f_{2}\right\|_{1}=\|P f\|_{1}
\end{aligned}
$$

and similarly for $P^{-1}$ so that

$$
\|f\|_{1}=\left\|P^{-1} P f\right\|_{1} \leq\|P f\|_{1} \leq\|f\|_{1}
$$

which gives the result.

Wendel (1952), Theorem 1 identified the multipliers of $L^{1}(G)$ as convolution operators, and also (Theorem 3) the isometric multipliers of $L^{1}(G)$ as those convolution operators generated by $\gamma \varepsilon_{x}$ where $x \in G$ and $\gamma \in \mathbf{C}$ with $|\gamma|=1$. The arguments used by Wendel carry over to hypergroups to give:

2.8. THEOREM. Let $K$ be a hypergroup and $T$ a bounded linear operator from $L^{1}(K)$ into itself. Then $T$ commutes with convolution if and only if there exists a unique measure $\mu \in M(K)$ such that $T f=\mu * f$ for each $f \in L^{1}(K)$.

2.9. THEOREM. Let $K$ be a hypergroup and consider the isometry $T: L^{1}(K)$ $\rightarrow L^{1}(K)$ given by $T f=\mu * f$. Then there exists $x \in K$ and $\gamma \in C$ with $|\gamma|=1$ such that $\mu=\gamma \varepsilon_{x}$.

It should be noted that in contrast to the group case the converse of Theorem 2.9 fails to hold. Indeed consider Example 9.1D of Jewett (1975) where 
the conjugacy class hypergroup of the alternating group on four letters is represented as $K=\{e, a, b, c\}$, with hypergroup dual $K^{-}=\{1, \chi, \psi, \xi\}$ related according to the following table:

\begin{tabular}{c|c|c|c|cc} 
& $e$ & $a$ & $b$ & $c$ \\
\hline 1 & 1 & 1 & 1 & 1 & \\
$\chi$ & 1 & 1 & $\alpha$ & $\beta$ & $\alpha=\bar{\beta}=e^{2 \pi i / 3}$. \\
$\psi$ & 1 & 1 & $\beta$ & $\alpha$ & \\
$\xi$ & 1 & $-1 / 3$ & 0 & 0
\end{tabular}

Then $T f=\varepsilon_{b} * f$ defines a multiplier of $L^{1}(K)$ into itself but $\varepsilon_{b} * \xi=0$ shows that $T$ is not norm preserving.

\section{Isomorphisms of $M(K)$}

Johnson (1964) metrically characterised the subspace of absolutely continuous measures in the measure algebra of a locally compact group to show that for locally compact groups $G_{1}, G_{2}, M\left(G_{1}\right)$ is isomorphic to $M\left(G_{2}\right)$ implies the same of $L^{1}\left(G_{1}\right)$ and $L^{1}\left(G_{2}\right)$. The same result was obtained independently by Strichartz (1965). We follow Strichartz' development which can be adapted to the hypergroup setting.

3.1. Lemma. For $\mu, \nu \in M(K)$

$$
\mu \perp \nu \text { if and only if }\|\mu+\nu\|=\|\mu-\nu\|=\|\mu\|+\|\nu\| .
$$

This lemma is proved in Strichartz (1965).

3.2. LeMMA. $L^{1}(K)$ is the intersection of all nontrivial closed left ideals I in $M(K)$ that satisfy

(3) $\mu \in I, \nu \in M(K)$ and $\nu \perp \lambda$ whenever $\mu \perp \lambda, \lambda \in M(K)$ imply $\nu \in I$.

Proof. We first see that $L^{1}(K)$ is a closed left ideal of $M(K)$ satisfying (3). Indeed that it is a closed left ideal is proved in Jewett (1975), Theorem 5.6B. Also for $\mu \in L^{1}(K)$ Strichartz (1965) showed that the condition on $\nu \in M(K)$ that $\nu \perp \lambda$ whenever $\mu \perp \lambda$ is equivalent to $\nu \ll \mu$; for if $\nu \ll \mu$ then there exists $g \in L^{1}(\mu)$ such that $\nu=g \mu$, and $\mu \perp \lambda$ implies $\nu \perp \lambda$. In the other direction if $\nu \perp \lambda$ whenever $\mu \perp \lambda$ then, in the 
Lebesgue decomposition $\nu=f \mu+\lambda$ we have as $\mu \perp \lambda$ that $\nu \perp \lambda$. Thus $\lambda=\nu-f \mu \perp \lambda$ and hence $\lambda=0$, so that $\nu=f \mu \ll \mu$. Condition (3) for $L^{1}(K)$ now follows as the Lebesgue-Radon-Nikodym theorem guarantees that $L^{1}(K)$ contains, along with each member, every measure that is absolutely continuous with respect to it.

It remains to show that any nontrivial closed left ideal $I$ of $M(K)$ satisfying (3) must contain $L^{1}(K)$. Indeed let $\mu \in I$ with $\mu \neq 0$. If $\left(V_{\alpha}\right)$ is a basis of compact neighbourhoods at $e$ and $k_{\alpha}=\omega\left(V_{\alpha}\right)^{-1} \xi_{V_{\alpha}}$ then $k_{\alpha} \in L^{1} \cap L^{\infty}(K)$, and for each $f \in C(K)$

$$
\begin{aligned}
\left|\int k_{\alpha} f d \omega-\int f d \varepsilon_{e}\right| & =\left|\omega\left(V_{\alpha}\right)^{-1} \int_{V_{\alpha}}(f-f(e)) d \omega\right| \\
& \leq \omega\left(V_{\alpha}\right)^{-1} \int_{V_{\alpha}}|f-f(e)| d \omega \\
& \leq \omega\left(V_{\alpha}\right)^{-1} \omega\left(V_{\alpha}\right) \varepsilon=\varepsilon
\end{aligned}
$$

for $\alpha$ sufficiently large, where we have used the continuity of $f$ at $e$. Thus $k_{\alpha} \rightarrow \varepsilon_{e}$ weakly, and from the continuity of the convolution, $k_{\alpha} * \mu \rightarrow \varepsilon_{e} * \mu=$ $\mu$. Since $\mu \neq 0$ there exists $\alpha$ for which $k_{\alpha} * \mu \neq 0$, and $\nu=k_{\alpha} * \mu \in L^{1}(K)$.

Now $|\nu| \ll \nu$ and hence by the first paragraph $|\nu| \in I$. Let $x \in \operatorname{supp}(|\nu|)$ and write $h_{\alpha}=\omega\left(\left\{x^{-}\right\} * V_{\alpha}\right)^{-1} \xi_{\left\{x^{-}\right\} * V_{\alpha}}$. Now by Jewett (1975), Lemma 4.2D

$$
\operatorname{pos}\left(h_{\alpha}\right) * \operatorname{supp}(|\nu|)=\operatorname{pos}\left(h_{\alpha} *|\nu|\right)
$$

where $\operatorname{pos}(h)=\{x: h(x)>0\}$. Now $x^{-} \in \operatorname{pos}\left(h_{\alpha}\right)$ and $x \in \operatorname{supp}(|\nu|)$ so that $e \in\left\{x^{-}\right\} *\{x\} \subset \operatorname{pos}\left(h_{\alpha} *|\nu|\right)$. Since $h_{\alpha} *|\nu| \in C(K)$ we see that $h_{\alpha} *|\nu|$ is strictly positive in a neighbourhood $V$ of $e$.

For $\alpha$ sufficiently large, $V_{\alpha} \subset V$ in which case $k_{\alpha} \omega \ll h_{\alpha} *|\nu|$. For such $\alpha$ we have $k_{\alpha} \omega \in I$, and since $I$ is a closed ideal and $\left(k_{\alpha}\right)$ is in fact an $L^{1}(K)$-bounded approximate identity it follows that $L^{1}(K) \subset I$.

We are now able to give the promised characterisation theorem which is an easy consequence of Lemmas 3.1 and 3.2.

3.3. THEOREM. Let $K_{1}, K_{2}$ be hypergroups, $T: M\left(K_{1}\right) \rightarrow M\left(K_{2}\right)$ an isometric isomorphism. Then $T$ restricted to $L^{1}\left(K_{1}\right)$ is an isometric isomorphism between $L^{1}\left(K_{1}\right)$ and $L^{1}\left(K_{2}\right)$.

Proof. Both $T$ and $T^{-1}$ preserve the property of being a closed left ideal. We also see that if $I$ is an ideal in $L^{1}\left(K_{1}\right)$ satisfying (3) then $T(I)$ 
also satisfies (3). Indeed for $T \mu \in T(I), T \nu \in M\left(K_{2}\right), T \nu \perp T \lambda$ whenever $T \mu \perp T \lambda$ (recall that $T$ is onto), and by Lemma 3.1

$$
\|T \nu+T \lambda\|=\|T \nu-T \lambda\|=\|T \nu\|+\|T \lambda\| .
$$

Since $T$ is an isometry this entails that

$$
\|\nu+\lambda\|=\|\nu-\lambda\|=\|\nu\|+\|\lambda\|
$$

so that $\nu \perp \lambda$ whenever $\mu \perp \lambda$. By (3), $\nu \in I$ and $T \nu \in T I$ as required. Similarly if $I$ is an ideal in $L^{1}\left(K_{2}\right)$ satisfying (3) then $T^{-1}(I)$ also satisfies (3).

It remains to observe that intersections are also preserved so that by Lemma 3.2, $T$ is an isometric isomorphism of $L^{1}\left(K_{1}\right)$ onto $L^{1}\left(K_{2}\right)$.

We can prove the converse of Theorem 3.3 by making use of Wendel's theorem for hypergroups.

3.4. TheOREM. Let $K_{1}, K_{2}$ be hypergroups, $T: L^{1}\left(K_{1}\right) \rightarrow L^{1}\left(K_{2}\right)$ an isometric isomorphism. Then $T$ extends to an isometric isomorphism between $M\left(K_{1}\right)$ and $M\left(K_{2}\right)$.

Proof. Denote by $\mathscr{L}\left(L^{1}\left(K_{i}\right), L^{1}\left(K_{i}\right)\right)$ the space of bounded linear operators from $L^{1}\left(K_{i}\right)$ into itself, $i=1,2$. Then $T$ extends to an isometric isomorphism $\mathrm{T}$ from $\mathscr{L}\left(L^{1}\left(K_{1}\right), L^{1}\left(K_{1}\right)\right)$ onto $\mathscr{L}\left(L^{1}\left(K_{2}\right), L^{1}\left(K_{2}\right)\right)$ via $\mathrm{T} S=T S T^{-1}$ where $S \in \mathscr{L}\left(L^{1}\left(K_{1}\right), L^{1}\left(K_{1}\right)\right)$. Also if $S$ commutes with convolution then so does $T S$, as can be seen from the chain of equalities

$$
\begin{aligned}
(\mathbf{T} S)(f * g) & =\left(T S T^{-1}\right)(f * g)=(T S)\left(T^{-1} f * T^{-1} g\right)=T\left[S\left(T^{-1} f\right) * T^{-1} g\right] \\
& =\left(T S T^{-1}\right)(f) * g=(\mathbf{T S})(f) * g .
\end{aligned}
$$

It remains to observe that $M\left(K_{i}\right)$ can be identified (isometrically) with the subspace of $\mathscr{L}\left(L^{1}\left(K_{i}\right), L^{1}\left(K_{i}\right)\right)$ consisting of those operators that commute with convolution; see Theorem 2.8 .

\section{Isomorphisms of hypergroups}

We have already observed that an isomorphism between two hypergroups is in fact an isomorphism between their measure algebras. However such a map does lead to a homeomorphism between the underlying locally compact spaces. We first need to identify the extreme points of the unit ball of $M(X)$, something that is well known for $X$ compact (see, for example, Dunford and 
Schwartz (1958), Lemma V.8.6, p. 441) or for real measures. However we can find no explicit reference to the more general result; our proof which is quite direct is included for completeness.

4.1. Lemma. Let $X$ be a locally compact Hausdorff space, $M(X)$ the set of complex Radon measures on $X$, and $B$ the closed unit ball in $M(X)$. Then $\operatorname{ext}(B)=\left\{\gamma \varepsilon_{x}: x \in X,|\gamma|=1\right\}$.

Proof. To show the forward inclusion we consider $\mu \in \operatorname{ext}(B)$. First note that $\|\mu\|=1$. Indeed if $\mu=0$ then $0=\frac{1}{2} \mu^{\prime}+\frac{1}{2}\left(-\mu^{\prime}\right)$ where $\left\|\mu^{\prime}\right\| \leq 1$ shows that 0 cannot be extremal. If $\mu \neq 0$ then $\mu=\|\mu\| \mu+(1-\|\mu\|) \mu$ would give the same conclusion if $\|\mu\|<1$.

Now suppose there exist $x, y \in \operatorname{supp}(\mu)$ with $x \neq y$. We can find an open neighbourhood $U$ of $x$ with $U^{c}$ disjoint from $y$; and $x \in \operatorname{supp}(\mu)$ shows that $|\mu|(U)>0$. Put $\mu_{1}=\left.\mu\right|_{U}$ and $\mu_{2}=\left.\mu\right|_{X \backslash U}$. Then $\mu=\mu_{1}+\mu_{2}$ and $\|\mu\|=\left\|\mu_{1}\right\|+\left\|\mu_{2}\right\|$. Thus

$$
\mu=\left\|\mu_{1}\right\| \frac{\mu_{1}}{\left\|\mu_{1}\right\|}+\left\|\mu_{2}\right\| \frac{\mu_{2}}{\left\|\mu_{2}\right\|}
$$

$(|\mu|(X \backslash U)>0$ as $y \in \operatorname{supp}(\mu))$. Also $\left\|\mu_{1}\right\|<\|\mu\| \leq 1$ and $\left\|\mu_{2}\right\|<\|\mu\| \leq 1$ shows that $\mu$ cannot be an extreme point of $B$. Hence $\operatorname{supp}(\mu)=\{x\}$ for some $x \in X$, and $\|\mu\|=1$ means that $\mu=\gamma \varepsilon_{x}$ for some $\gamma$ with $|\gamma|=1$.

For the reverse inclusion, let $x \in X,|\gamma|=1$ and suppose $\gamma \varepsilon_{x}=b \mu_{1}+$ $(1-b) \mu_{2}$ for some $\mu_{1}, \mu_{2} \in B, 0<b<1$. Clearly $\left\|\mu_{1}\right\|=\left\|\mu_{2}\right\|=1$. We want to show that $\gamma \varepsilon_{x}=\mu_{1}=\mu_{2}$.

We first show that if $h \in C_{c}(X)$ vanishes on an open neighbourhood $U$ of $x$ then

$$
\int_{X} h d \mu_{1}=\int_{X} h d \mu_{2}=0 .
$$

We can assume without loss of generality that $\|h\|_{\infty} \leq 1$. Since $X$ is a $T 3 \frac{1}{2}$ space there exists $k \in C(X)$ such that $\|k\|_{\infty} \leq 1, k(x)=1$ and $k=0$ outside $U$. In fact by choosing such $k$ with respect to a relatively compact open subset of $U$ containing $x$ we can further assume that $k$ has compact support. Now

$$
b \int_{X} k d \mu_{1}+(1-b) \int_{X} k d \mu_{2}=\gamma \int_{X} k d \varepsilon_{x}=\gamma k(x)=\gamma .
$$

But $|\gamma|=1$ gives that $\gamma$ is an extreme point of the closed unit ball in $\mathbf{C}$. Since

$$
\left|\int_{X} k d \mu_{i}\right| \leq\|k\|_{\infty}\left\|\mu_{i}\right\| \leq 1
$$


for $i=1,2$, we can deduce that

$$
\gamma=\int_{X} k d \mu_{1}=\int_{X} k d \mu_{2}
$$

Similarly $\|h+k\|_{\infty} \leq 1$ gives the same equalities with $h+k$ replacing $k$ so that

$$
\int_{X} h d \mu_{1}=\int_{X} h d \mu_{2}=0 .
$$

This shows that $\mu_{1}, \mu_{2}$ vanish on $X \backslash\{x\}$ and hence $\operatorname{supp}\left(\mu_{1}\right)=\operatorname{supp}\left(\mu_{2}\right)=$ $\{x\}$. Thus $\mu_{1}=\gamma_{1} \varepsilon_{x}$ and $\mu_{2}=\gamma_{2} \varepsilon_{x}$ for some $\gamma_{1}, \gamma_{2}$ with $\left|\gamma_{1}\right|=\left|\gamma_{2}\right|=1$; and

$$
\gamma \varepsilon_{x}=b \gamma_{1} \varepsilon_{x}+(1-b) \gamma_{2} \varepsilon_{x}=\left(b \gamma_{1}+(1-b) \gamma_{2}\right) \varepsilon_{x}
$$

gives $\gamma=b \gamma_{1}+(1-b) \gamma_{2}$. Thus $\gamma=\gamma_{1}=\gamma_{2}$ as $\gamma$ is extremal in the closed unit ball of $\mathrm{C}$, and $\mu_{1}=\mu_{2}=\gamma \varepsilon_{x}$ as required.

Now suppose that $T: M\left(K_{1}\right) \rightarrow M\left(K_{2}\right)$ is an isometric isomorphism. Then $T\left(B_{1}\right)=B_{2}$, where $B_{i}$ denotes the closed unit ball of $M\left(K_{i}\right), i=$ 1, 2. Clearly $T\left(\operatorname{ext}\left(B_{1}\right)\right)=\operatorname{ext}\left(B_{2}\right)$. Indeed if $\mu \in \operatorname{ext}\left(B_{1}\right)$ and $T \mu=\alpha \nu_{1}+$ $(1-\alpha) \nu_{2}$ where $0 \leq \alpha \leq 1, \nu_{1}, \nu_{2} \in B_{2}$ then $\mu=\alpha T^{-1} \nu_{1}+(1-\alpha) T^{-1} \nu_{2}$, and $\left\|T^{-1} \nu_{1}\right\| \leq 1,\left\|T^{-1} \nu_{2}\right\| \leq 1$ shows that $\mu=T^{-1} \nu_{1}=T^{-1} \nu_{2}$ and $T \mu=\nu_{1}=\nu_{2}$. Thus $T \mu \in \operatorname{ext}\left(B_{2}\right)$ and $T\left(\operatorname{ext}\left(B_{1}\right)\right) \subset \operatorname{ext}\left(B_{2}\right)$. Since $T^{-1}$ is also an isometric isomorphism we have $T^{-1}\left(\operatorname{ext}\left(B_{2}\right)\right) \subset \operatorname{ext}\left(B_{1}\right)$, and these combine to give the desired equality.

The above paragraph shows that for each $x \in K_{1}$ there exists $y \in K_{2}$ such that $T \varepsilon_{x}=\gamma(x) \varepsilon_{y}$ where $|\gamma(x)|=1$. We write $y=\tau(x)$. As can be expected $\tau$ preserves some of the structure of $K_{1}$ and $K_{2}$. We first need a result on the vague convergence of measures.

4.2. Theorem. (a) If $\left(\mu_{\alpha}\right)$ is norm bounded in $M(K)$ and $\|\left(\mu_{\alpha}-\mu\right) *$ $f \|_{1} \rightarrow 0$ for all $f \in L^{1}(K)$ then $\mu_{\alpha} \rightarrow \mu$ vaguely.

(b) If $\mu_{\alpha}, \mu \in M^{+}(K)$ with $\mu_{\alpha} \rightarrow \mu$ then $\left\|\left(\mu_{\alpha}-\mu\right) * f\right\|_{1} \rightarrow 0$ for all $f \in L^{1}(K)$.

Proof. (a) For $f \in L^{1}(K)$ and $g \in C_{0}(K)$ we have using Jewett (1975), Lemma $4.2 \mathrm{H}$

$$
\int f * g^{-} d\left(\mu_{\alpha}^{-}-\mu^{-}\right)=\int g * f^{-} d\left(\mu_{\alpha}-\mu\right)=\int\left(\mu_{\alpha}-\mu\right) * f g d \omega \rightarrow 0
$$

by the assumption on $\left(\mu_{\alpha}\right)$. Now $L^{1} * C_{0}(K)$ is dense in $C_{0}(K)$; indeed if $\left(k_{\beta}\right)$ is the bounded approximate unit in $L^{1}(K)$ given in the proof of 
Lemma 3.2 then $k_{\beta} \rightarrow \varepsilon_{e}$ weakly, and appealing to Jewett (1975), Lemma $4.2 \mathrm{~F}$ for $g \in C_{0}(K)$ we have

$$
\lim _{\beta}\left\|k_{\beta} * g-\varepsilon_{e} * g\right\|_{\infty}=0
$$

so that $k_{\beta} * g \in L^{1} * C_{0}(K)$ approximates $g$.

We now use the norm boundedness of $\left(\mu_{\alpha}\right)$ and (4) to deduce that $\mu_{\alpha} \rightarrow \mu$ on $C_{0}(K)$ as required.

(b) To prove this we follow the proof of Theorem $\mathrm{D}$ (a) given in Granirer and Leinert (1981). The only part that needs attention is the property that $\mu_{\alpha} * f \rightarrow \mu * f$ uniformly on each compact set for $f \in C_{c}(K)$. But this has been proved in Bloom and Heyer (1989), Theorem 2.9, and the result is complete.

4.3. CoRollary. Every hypergroup $K$ is homeomorphic to $\left\{\varepsilon_{x}: x \in K\right\}$ where the latter is given the point-norm convergence topology as a space of operators on $L^{1}(K)$; that is $x_{\alpha} \rightarrow x$ if and only if $\left\|\varepsilon_{x_{\alpha}} * f-\varepsilon_{x} * f\right\|_{1} \rightarrow 0$ for all $f \in L^{1}(K)$.

4.4. ThEOREM. The isometric isomorphism $T: M\left(K_{1}\right) \rightarrow M\left(K_{2}\right)$ induces a homeomorphism $\tau: K_{1} \rightarrow K_{2}$ that preserves compact subhypergroups.

Proof. First note that $\tau$ is one-to-one as $\tau\left(x_{1}\right)=\tau\left(x_{2}\right)$ implies $\bar{\gamma}\left(x_{1}\right) T \varepsilon_{x_{1}}$ $=\bar{\gamma}\left(x_{2}\right) T \varepsilon_{x_{2}}$ and $T \varepsilon_{x_{1}}=T\left(\gamma\left(x_{1}\right) \bar{\gamma}\left(x_{2}\right) \varepsilon_{x_{2}}\right)$. Since $T$ is one-to-one $\varepsilon_{x_{1}}=$ $\gamma\left(x_{1}\right) \bar{\gamma}\left(x_{2}\right) \varepsilon_{x_{2}}$, and considering the supports of these two measures we have $x_{1}=x_{2}$.

To show that $\tau$ is onto choose $y \in K_{2}$ and, since $T^{-1}: M\left(K_{2}\right) \rightarrow M\left(K_{1}\right)$ is an isometric isomorphism, $x \in K_{1}$ and $\alpha_{y}$ with $\left|\alpha_{y}\right|=1$ such that $T^{-1} \varepsilon_{y}=\alpha_{y} \varepsilon_{x}$. Then

$$
\gamma(x) \varepsilon_{\tau(x)}=T \varepsilon_{x}=T\left(\bar{\alpha}_{y} T^{-1} \varepsilon_{y}\right)=\bar{\alpha}_{y} \varepsilon_{y}
$$

and again considering supports we have $y=\tau(x)$ (and $T^{-1} \varepsilon_{\tau(x)}=\bar{\gamma}(x) \varepsilon_{x}$ ). Consider $x_{\alpha} \rightarrow x$ so that by Corollary $4.3, \varepsilon_{x_{\alpha}} \rightarrow \varepsilon_{x}$ in the point-norm convergence topology. Since $T: M\left(K_{1}\right) \rightarrow M\left(K_{2}\right)$ is a norm-preserving algebra isomorphism we have $\left\|T \varepsilon_{x_{\alpha}} * T f-T \varepsilon_{x} * T f\right\|_{1} \rightarrow 0$. Now appeal to Theorem 3.3 to have that $T$ restricted to $L^{1}\left(K_{1}\right)$ is an isometric isomorphism between $L^{1}\left(K_{1}\right)$ and $L^{1}\left(K_{2}\right)$ so that $\left\|T \varepsilon_{x_{\alpha}} * g-T \varepsilon_{x} * g\right\|_{1} \rightarrow 0$ for all $g \in L^{1}\left(K_{2}\right)$. By Theorem 4.2(a)

$$
\gamma\left(x_{\alpha}\right) \varepsilon_{\tau\left(x_{\alpha}\right)}=T \varepsilon_{x_{\alpha}} \rightarrow T \varepsilon_{x}=\gamma(x) \varepsilon_{\tau(x)}
$$


in the vague topology. In particular it must be the case that $\tau\left(x_{\alpha}\right) \rightarrow \tau(x)$ so that $\tau$ is continuous. Similarly from $T^{-1} \varepsilon_{y}=\bar{\gamma}\left(\tau^{-1}(y)\right) \varepsilon_{\tau^{-1}(y)}$ we see that $\tau^{-1}$ is also continuous. Thus $\tau$ is a homeomorphism.

Let $H$ be a compact subhypergroup of $K_{1}$ and write $\omega_{H}$ for its normalised Haar measure. Then $\omega_{H}$ is idempotent and

$$
T \omega_{H}=T\left(\omega_{H} * \omega_{H}\right)=T \omega_{H} * T \omega_{H}
$$

shows that $T \omega_{H}$ is idempotent. We now make use of Lemma 4.5 below to write $\tau(H)=\tau\left(\operatorname{supp}\left(\omega_{H}\right)\right)=\operatorname{supp}\left(T \omega_{H}\right)$ which by the continuity of $\tau$ must be compact. We also have by Jewett (1975), Lemma 3.2G that

$$
\begin{aligned}
\tau(H) * \tau(H) & =\operatorname{supp}\left(T \omega_{H}\right) * \operatorname{supp}\left(T \omega_{H}\right) \\
& =\operatorname{supp}\left(T \omega_{H} * T \omega_{H}\right) \\
& =\operatorname{supp}\left(T \omega_{H}\right)=\tau(H)
\end{aligned}
$$

and from Corollary 4.6 below $\tau(H)^{-}=\tau\left(H^{-}\right)=\tau(H)$. Now appeal to Jewett (1975), Theorem $10.2 \mathrm{~F}$ to deduce that $\tau(H)$ is a compact subhypergroup of $K$.

We can show that $\gamma$ has many of the properties of a character of $K_{1}$. We first prove a preliminary result.

\subsection{Lemma. For each $\mu \in M_{c}^{+}\left(K_{1}\right), \tau(\operatorname{supp}(\mu))=\operatorname{supp}(T \mu)$.}

Proof. We first note that $T$ is weakly continuous on $M^{+}(K)$ in the sense that if $\mu_{\alpha} \rightarrow \mu$ then $T \mu_{\alpha} \rightarrow T \mu$ vaguely. Indeed by Theorem 4.2(b) for such a net $\left(\mu_{\alpha}\right),\left\|\left(\mu_{\alpha}-\mu\right) * f\right\|_{1} \rightarrow 0$ for all $f \in L^{1}\left(K_{1}\right)$, and as in the proof of Theorem 4.4, $\left\|\left(T \mu_{\alpha}-T \mu\right) * g\right\|_{1} \rightarrow 0$ for all $g \in L^{1}\left(K_{2}\right)$ so that (note that $\left(\mu_{\alpha}\right)$ must be norm bounded) by Theorem $4.2(\mathrm{a}), T \mu_{\alpha} \rightarrow T \mu$ vaguely.

Now we use the property that every positive measure $\mu$ with compact support can be approximated by a positive discrete measure $\nu$ with $\operatorname{supp}(\nu) \subset$ $\operatorname{supp}(\mu)$ and $\|\nu\|=\|\mu\|$. From $T \varepsilon_{x}=\gamma(x) \varepsilon_{\tau(x)}$ we have

$$
T \mu=T\left(\int \varepsilon_{x} d \mu(x)\right)=\int T \varepsilon_{x} d \mu(x)=\int \gamma(x) \varepsilon_{\tau(x)} d \mu(x)
$$

where we have used the weak continuity of $T$ proved above. Then choosing $f \in C\left(K_{2}\right)$ with support disjoint from $\tau(\operatorname{supp}(\mu))$ we have

$$
T \mu(f)=\int \gamma(x) f(\tau(x)) d \mu(x)=0
$$

so that $\operatorname{supp}(T \mu) \subset \tau(\operatorname{supp}(\mu))$. 
In the other direction, we first note that $\gamma$ is continuous on $K_{1}$, which follows immediately from the definition of $\gamma$ and the continuity of $\tau$. Choose $y \in \tau(\operatorname{supp}(\mu))$, so that $y=\tau\left(x_{0}\right)$ for some $x_{0} \in \operatorname{supp}(\mu)$, and a neighbourhood $V$ of $x_{0}$. Then there exists $g \in C^{+}\left(K_{1}\right)$ supported in $V$ such that $\mu(g) \neq 0$. Now $f=g \circ \tau^{-1} \in C^{+}\left(K_{2}\right)$, and from the continuity of $\gamma$

$$
T \mu(f)=\int \gamma(x) f(\tau(x)) d \mu(x) \neq 0
$$

provided $V$ is taken suitably small. Thus $y \in \operatorname{supp}(T \mu)$ and this completes the proof.

4.6. CoRollary. $\tau(\{x\} *\{y\})=\{\tau(x)\} *\{\tau(y)\}$ and $\tau\left(x^{-}\right)=\tau(x)^{-}$for all $x, y \in K_{1}$.

Proof. Using Lemma 4.5 we have for $x, y \in K_{1}$

$$
\begin{aligned}
\tau(\{x\} *\{y\}) & =\tau\left(\operatorname{supp}\left(\varepsilon_{x} * \varepsilon_{y}\right)\right)=\operatorname{supp}\left(T\left(\varepsilon_{x} * \varepsilon_{y}\right)\right) \\
& =\operatorname{supp}\left(T \varepsilon_{x} * T \varepsilon_{y}\right)=\operatorname{supp}\left(\gamma(x) \gamma(y) \varepsilon_{\tau(x)} * \varepsilon_{\tau(y)}\right) \\
& =\{\tau(x)\} *\{\tau(y)\}
\end{aligned}
$$

For the second equality just take $y=x^{-}$to give $\tau\left(\{x\} *\left\{x^{-}\right\}\right)=\{\tau(x)\} *$ $\left\{\tau\left(x^{-}\right)\right\}$. Then $e_{1} \in\{x\} *\left\{x^{-}\right\}$gives $e_{2}=\tau\left(e_{1}\right) \in\{\tau(x)\} *\left\{\tau\left(x^{-}\right)\right\}$and hence $\tau\left(x^{-}\right)=\tau(x)^{-}$.

4.7. TheOREM. The function $\gamma$ satisfies $\gamma(x * y)=\gamma(x) \gamma(y)$ for all $x, y \in$ $K_{1}$. If furthermore $T \mu^{-}=(T \mu)^{\sim}$ for all $\mu \in M^{1}\left(K_{1}\right)$ then $\gamma$ is a character of $K_{1}$.

Proof. Let $x, y \in K_{1}$. Then from the weak continuity of $T$

$$
\begin{gathered}
\gamma(x) \gamma(y) \varepsilon_{\tau(x)} * \varepsilon_{\tau(y)}=T\left(\varepsilon_{x} * \varepsilon_{y}\right)=T\left(\int \varepsilon_{s} d \varepsilon_{x} * \varepsilon_{y}(s)\right) \\
=\int T \varepsilon_{s} d \varepsilon_{x} * \varepsilon_{y}(s)=\int \gamma(s) \varepsilon_{\tau(s)} d \varepsilon_{x} * \varepsilon_{y}(s) .
\end{gathered}
$$

Choose $f \in C\left(K_{2}\right)$ such that $f=1$ on $\tau(\{x\} *\{y\})$. Then from Corollary 4.6

$$
\begin{aligned}
\gamma(x * y) & =\int \gamma(s) \varepsilon_{\tau(s)}(f) d \varepsilon_{x} * \varepsilon_{y}(s) \\
& =\gamma(x) \gamma(y) \varepsilon_{\tau(x)} * \varepsilon_{\tau(y)}(f)=\gamma(x) \gamma(y) .
\end{aligned}
$$

If $T \mu^{-}=(T \mu)^{\sim}$ for all $\mu \in M^{1}\left(K_{1}\right)$ then

$$
\gamma\left(x^{-}\right) \varepsilon_{\tau\left(x^{-}\right)}=T \varepsilon_{x^{-}}=\left(T \varepsilon_{x}\right)^{\sim}=\overline{\gamma(x)} \varepsilon_{\tau(x)^{-}} .
$$

Thus $\gamma\left(x^{-}\right)=\overline{\gamma(x)}$. 


\section{References}

Wolfgang Arendt and Jean de Cannière, 'Order isomorphisms of Fourier algebras,' J. Funct. Anal. 50 (1983), 1-7.

Walter R. Bloom, 'Idempotent measures on commutative hypergroups,' Probability Measures on Groups VIII (Proc. Conf., Oberwolfach Math. Res. Inst., Oberwolfach, 1985), Lecture Notes in Math. 1210, Springer-Verlag, Berlin, Heidelberg, New York, London, Paris, Tokyo 1986, 13-23.

Walter R. Bloom and Herbert Heyer, 'Convergence of convolution products of probability measures on hypergroups,' Rend. Mat. Ser. VII 3 (1982), 547-563.

Walter R. Bloom and Herbert Heyer, 'Characterisation of potential kernels of transient convolution semigroups on a commutative hypergroup,' Probability Measures on Groups IX (Proc. Conf., Oberwolfach Math. Res. Inst., Oberwolfach 1988), Lecture Notes in Math. 1379, Springer-Verlag, Berlin, Heidelberg, New York, London, Paris, Tokyo, 1989, pp. 21-35.

Nelson Dunford and Jacob T. Schwartz, Linear Operators, Part 1: General Theory, Interscience Publishers, New York, London, 1958.

Charles F. Dunkl, 'The measure algebra of a locally compact hypergroup' Trans. Amer. Math. Soc. 179 (1973), 331-348.

E. E. Granirer and M. Leinert, 'On some topologies which coincide on the unit sphere of the Fourier-Stieltjes algebra $B(G)$ and of the measure algebra $M(G)$, Rocky Mountain $J$. Math. 11 (1981), 459-472.

Robert I. Jewett, 'Spaces with an abstract convolution of measures,' Adv. in Math. 18 (1975), 1-101.

B. E. Johnson, 'Isometric isomorphisms of measure algebras,' Proc. Amer. Math. Soc. 15 (1964), $186-188$.

Yukiyosi Kawada, 'On the group ring of a topological group,' Math. Jap. 1 (1948), 1-5.

Kenneth A. Ross, 'Hypergroups and centers of measure algebras,' Symp. Math. 22 (1977), 189203.

R. Spector, 'Aperçu de la théorie des hypergroupes,' Analyse Harmonique sur les Groupes de Lie (Séminaire Nancy-Strasbourg, 1973-1975), Lecture Notes in Math. 497, SpringerVerlag, Berlin, Heidelberg, New York, 1975, 643-673.

R. Spector. 'Mesures invariantes sur les hypergroupes,' Trans. Amer. Math. Soc. 239 (1978), 147-165.

Robert S. Strichartz, 'Isometric isomorphisms of measure algebras,' Pacific J. Math. 15 (1965), 315-317.

Martin E. Walter, ' $W^{*}$-algebras and nonabelian harmonic analysis,' J. Funct. Anal. 11 (1972), 17-38.

J. G. Wendel, 'On isometric isomorphisms of group algebras,' Pacific J. Math. 1 (1951), 305-311.

J. G. Wendel, 'Left centralizers and isomorphisms of group algebras,' Pacific J. Math. 2 (1952), 251-261.

School of Mathematical and Physical

Sciences

Murdoch University

Perth

Western Australia 6150

Australia
Department of Mathematics University of Colorado Campus Box 426, Boulder Colorado 80309

USA 